\title{
Rapunzelsyndrom - wenn das Haar den Darm verstopft
}

\author{
Manche Menschen verspüren einen starken Drang, das \\ eigene Haar zu verspeisen. Dies kann sich im Magen \\ und Darm ansammeln und einen Darmverschluss mit \\ drastischen Folgen auslösen.
}

„Rapunzel, Rapunzel, lass dein Haar herunter - aber doch bitte nicht in den Rachen!“, möchte man bei Patienten mit Rapunzelsyndrom ergänzen. Die Betroffenen ziehen sich nicht nur die Haare aus der Kopfhaut (Trichotillomanie), sondern verschlucken sie auch noch (Trichophagie).

Bleibt die Hauptmasse als Bezoar im Magen liegen, wobei sich Haarsträhnen bis in den Dünndarm oder im Extremfall durch den gesamten Dickdarm ziehen, ähnlich wie die Locken der Märchenschönheit vom Turmkerker bis in die Hände des Prinzen, dann spricht man vom Rapunzelsyndrom. Doch für die Betroffenen hat das Syndrom nichts Romantisches - wie es der Fall einer Patientin mit besonders großem Appetit auf die eigenen Haare illustriert.

\section{Deutliche Hypoproteinämie}

Über die 38-jährige Patientin berichten Ärzte um Dr. Waqas Ullah von der Uniklinik in Tucson. Sie war zuvor noch nie psychiatrisch auffällig gewesen und stellte sich mit seit zwei Tagen anhaltendem Erbrechen, ausgeprägter Verstopfung und starken Blähungen vor. Das Erbrochene war fäkaloid und von wässriger Konsistenz. In der Anamnese verneinte die Frau Fieber, Bauchschmerzen, Hämoptoe oder Hämatemesis. Auffällig war jedoch ein Gewichtsverlust von rund $8 \mathrm{~kg}$ in den vergangenen acht Monaten. Zudem beschrieb sie einen Mangel an Appetit, der ein Jahr zuvor eingesetzt habe.

Die Frau wirkte lethargisch und war sehr blass, der Bauch deutlich aufgetrieben, jedoch ohne tastbare Auffälligkeiten. An den Beinen hatte die Patientin beidseitig deutliche eindrückbare Ödeme. Die kardiovaskuläre und neurologische Untersuchung waren unauffällig.

Laborwertuntersuchungen ergaben erniedrigte Hämoglobin- und MCV-Werte bei normaler Plättchen- und Leukozyten- zahl. Mit 6 g/dl war auch der Serumproteinwert unter der Norm, ebenso der Serumalbumin- (2,5 g/dl) sowie der Natriumund Kaliumspiegel.

Im Röntgenbild nach Bariumaufnahme zeigten sich erweiterte Darmschlingen Haustren waren kaum zu erkennen. Die Ärzte tippten auf einen paralytischen Ileus und eine Obstruktion durch eine abdominale Masse.

Da sich der Zustand der Patientin fortwährend verschlechterte, wurde sie schließlich einer Operation unterzogen. Dabei fanden die Ärzte ein 15 x $10 \mathrm{~cm}$ großes Haarknäul im Magen mit Ausläufern bis ins Duodenum und zusätzlich ein $4 \times 3 \mathrm{~cm}$ messendes Knäuel im distalen Ileum. Sechs Tage nach chirurgischer Entfernung der Haare aus Magen und Darm konnte die Frau entlassen und an einen Psychiater überwiesen werden.

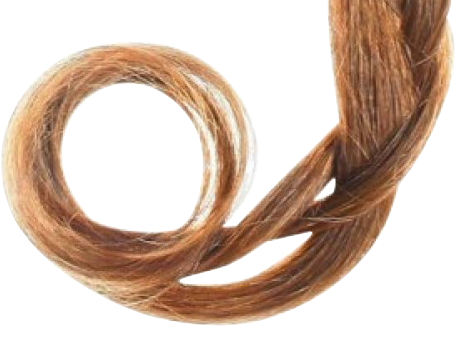

Schätzungsweise 1-4\% der Bevölkerung hat eine Neigung, sich die eigene Haare auszureißen, bis zu 20\% davon verschlingen sie auch, und von ihnen entwickelt wiederum ein Drittel ein Rapunzelsyndrom. Häufige Symptome sind Erbrechen, Appetitverlust und Blähungen.

Das Rapunzelsyndrom geht nicht selten mit Depressionen, geistiger Retardierung, Bulimie, Persönlichkeitsstörung oder Schizophrenie einher. Die Betroffenen sind meist jung.

(Thomas Müller)

Ullah Wet al. BMJ 2016, ePub 26.9.2016, doi:10.1136/ bcr-2016-216600

\section{Schlaf- und Lebensqualität verbessern}

\section{ALS: Nächtliche Beatmung verdoppelt Lebenszeit}

\section{ALS-Patienten leiden häufig unter} schlechtem Schlaf. Die nichtinvasive Beatmung bessert nicht nur Schlaf und Lebensqualität - sie verlängert das Leben deutlich.

Ein schlechter Schlaf verursacht bei Patienten mit amyotropher Lateralsklerose (ALS) mit den höchsten Leidensdruck. Gestört wird der Schlaf meist durch Faszikulationen, Krämpfe, Hypoventilation oder Schmerzen. Ein Teil der Probleme lässt sich auf die nächtliche Hypoventilation der Betroffenen zurückführen. Mit ei- ner nichtinvasiven Beatmung lasse sich der Schlaf oft wieder normalisieren, so Privatdozent Matthias Boentert, Münster. Doch nicht nur Schlaf- und Lebensqualität der Patienten profitieren davon. Boentert zitierte Studien, nach denen auch die Lebenszeit durch die nächtliche Beatmung deutlich verlängert wird. So lebten in einer Studie mit knapp 1000 ALS-Patienten solche mit Beatmung im Schnitt noch 29, die ohne nur 15 Monate.

(mut)

89. Kongress der Deutschen Gesellschaft für Neurologie (DGN), Mannheim, September 2016 